\title{
Medication-related osteonecrosis of the jaw, osteoradionecrosis, and osteomyelitis: A comparative histopathological study
}

\section{Carlos Cesar DE ANTONI(a) Mariza Akemi MATSUMOTO(b) Andréia Aparecida da SILVA ${ }^{(a)}$ Marcos Martins CURI(c) Joel Ferreira SANTIAGO JÚNIOR(a) Laurindo Moacir SASSI(d) Camila Lopes CARDOSO(a)}

\footnotetext{
(a) Universidade do Sagrado Coração, Department of Oral Surgery, Bauru, SP, Brazil.

(b) Universidade Estadual Paulista, Department of Histology, Araçatuba, SP, Brazil.

(c) Hospital Santa Catarina, Department of Stomatology, São Paulo, SP, Brazil.

(d) Hospital Erasto Gaertner, Department of Oral Surgery, Curitiba, PR, Brazil.
}

Declaration of Interests: The authors certify that they have no commercial or associative interest that represents a conflict of interest in connection with the manuscript.

Corresponding Author:

Camila Lopes Cardoso

E-mail:cardoso_lopes@yahoo.com.br

Submitted: September 19, 2017

Accepted for publication: January 31, 2018

Last revision: February 16, 2018

\begin{abstract}
It is largely known that some oral diseases can be diagnosed based upon their clinical manifestation combined with the patient's medical history and generally not depending on histopathological examination. This is the case of some bone diseases such as osteoradionecrosis of the jaw (ORNJ), osteomyelitis of the jaw (OMJ), and medication-related osteonecrosis of the jaw (MRONJ). The present study aimed to analyze the histopathological features of these specific bone diseases in order to evaluate similarities and differences. Forty-four bone specimens resected from each bone disease (22 cases of ORNJ, 6 cases of OMJ, and 16 cases of MRONJ) were analyzed by two experienced oral pathologists without prior knowledge of the diagnosis, considering bone tissue condition, inflammation, vascularization, and the presence of microorganisms. In addition, the examiners formulated a diagnostic hypothesis for each specimen. Many histopathological similarities were found among the diseases, especially considering the presence of necrotic bone, inflammation, and microorganisms. Statistically significant differences were detected in empty bone lacunae, which was decreased in ORN ( $p=0.042)$, and considering neutrophil count, which was low in the MRONJ group $(\mathrm{p} \leq 0.001)$. The Kappa coefficient was calculated and agreement was detected based on the histopathological parameters, but not for diagnostic suggestion $(p=0.23)$. In conclusion, histopathological aspects of ORNJ, OMJ, and MRONJ do not permit a conclusive diagnosis, emphasizing the necessity of a detailed clinical report.
\end{abstract}

Keywords: Osteonecrosis; Osteomyelitis; Osteoradionecrosis.

\section{Introduction}

Histopathological analysis may be critical for confirming the diagnosis of many craniomaxillofacial diseases. However, ideally, the combination of clinical findings and complementary exams should be followed, since specific conditions such as those affecting bone tissue may present quite similar histological features. Among them, particular attention is given to osteomyelitis (OMJ), osteoradionecrosis (ORNJ), and medication-related osteonecrosis of the jaw (MRONJ)., ${ }^{1,2,3}$ However, it is important to emphasize 
that these are quite distinct clinical pathologies with different etiologies. ${ }^{2}$

Osteomyelitis (OMJ) is a bone pathology which generally occurs due to complications arising from an acute dentoalveolar abscess and is caused by the microbial infection with eventual tissue destruction and necrosis, extending beyond the initial area of involvement. ${ }^{4}$ Regarding bone necrosis, osteoradionecrosis of the jaw (ORNJ) is a common and serious complication following head and neck radiation therapy, ${ }^{5}$ and medication-related osteonecrosis (MRONJ) is considered the most common complication associated with bisphosphonate therapy. ${ }^{6}$

Bone necrosis is a common characteristic of these bone diseases, which also have clinical signs and symptoms. ${ }^{2}$ Despite histopathological and clinical similarities, the above-mentioned pathologies also exhibit very similar image characteristics. ${ }^{7,8}$ Authors have presented a detailed image description comparing BRONJ and ORNJ from dental panoramic radiographs and computed tomography and have pointed out a number of similarities between the two pathologies, such as osteolysis, osteosclerosis, sequestration, and dissemination of soft tissue inflammation, emphasizing only two main differences between them, namely predominant osteolysis in ORNJ and osteosclerosis in MRONJ cases.7

There are few pathological studies comparing OMJ, MRONJ, and ORNJ in the literature. ${ }^{1,23,9}$ Therefore, the present study aimed to compare the histopathological features of these bone diseases in order to detect some common or prevalent aspects.

\section{Methodology}

A retrospective histopathological evaluation was performed on specimens selected from patients' records from 2000 to 2017, previously subjected to biopsy of the maxillary bones and diagnosed as OMJ, ORNJ, and MRONJ associated with bisphosphonate. The final diagnosis of each disease was recorded in the patients' medical records and it was based on clinical, radiographic, and microscopic aspects, combined with the medical history. This study was approved by the institutional review board (process no. 1.249.820). The patients' records were reviewed and the following clinical criteria were used for the diagnosis of OMJ: presence of bone infection, suppuration and radiographic evidence of osteolysis, absence of head and neck radiation therapy, and no use of bisphosphonates. ${ }^{2,4}$ The diagnostic criteria for MRONJ were the presence of exposed necrotic bone in the jaws for at least 8 weeks associated with bisphosphonate use and absence of head and neck radiation therapy. ${ }^{2,6}$ The diagnostic criteria for ORNJ were the presence of exposed necrotic bone in the jaws for at least 8 weeks in a patient who received at least $50 \mathrm{~Gy}$ of head and neck radiation therapy and absence of bisphosphonate therapy. ${ }^{5}$

The specimens were obtained from existing paraffin-embedded blocks of each bone disease from three different institutions. Microscope slides were cut in 6- $\mu \mathrm{m}$ sections and stained with hematoxylin and eosin (HE), and analyzed by two experienced oral pathologists, who were blinded to the diagnosis. The following parameters were considered for the histopathological analysis: overall bone tissue status (vitality, necrosis, reactivity), bone cells (osteoblasts, osteoclasts), inflammation, vascularization (blood vessels, thrombosis), and presence of biofilm. The detailed criteria are displayed in Table 1, where the examiners marked the absence or presence of each item, and at the end, each pathologist made a presumptive diagnosis, limited to the three diseases. After the evaluation, the diagnoses were compared with the original ones.

Table 1. Microscopic evaluation criteria.

\begin{tabular}{lc}
\hline & Necrotic bone \\
& Reactive bone \\
Bone evaluation & Osteoclast \\
& Osteoblast \\
& Empty osteocyte lacunae \\
Inflammation & Lymphocytes \\
& Macrophages \\
Vascularization & Neutrophils \\
& Blood vessels \\
\hline Microorganisms & Hyperemia and thrombosis \\
\hline
\end{tabular}



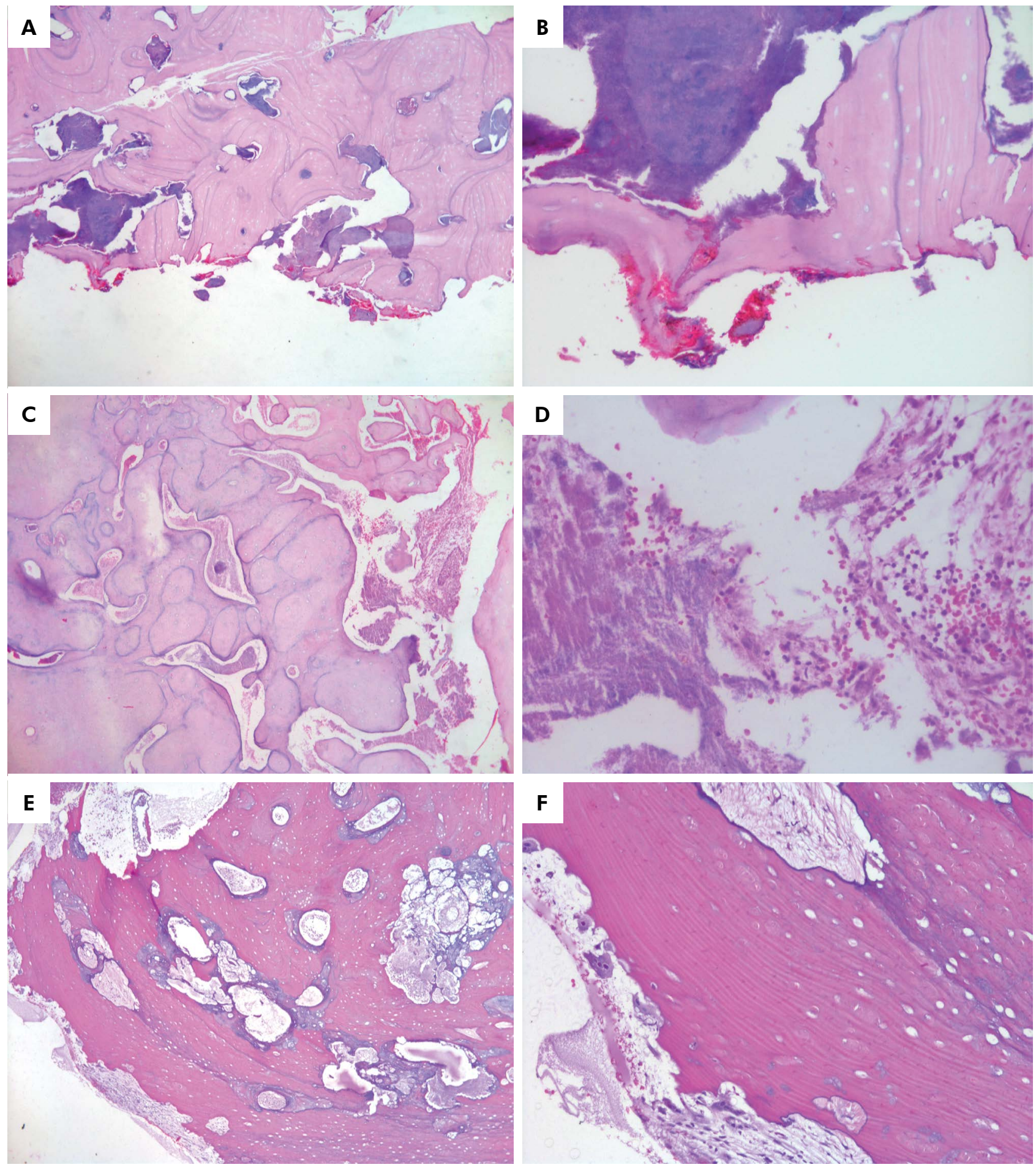

Figure 1. $a, b)$ MRONJ - extensive biofilms on the surfaces of non-viable bone tissue; c) OMJ - Non-viable bone tissue presenting basophilic reversal lines, d) biofilm with leukocyte infiltration; and, e, f) ORNJ - Avascular and acellular bone tissue showing areas with osteoclastic activity (HE; bar $=100 \mu \mathrm{m})$.

The data were fed into an Excel spreadsheet (Microsoft Office Excel, Redmond, WA, USA), analyzed by SigmaPlot software (SigmaPlot, San Jose, CA,
USA) version 12.3 , and their normal distribution and equal variance were checked by the Shapiro-Wilk test, followed by the Kruskal-Wallis test (one-way 
analysis of variance on ranks (variable: Diagnosis)) and Dunn's post-hoc test, in order to verify the differences between groups at a $5 \%$ significance level. The agreement between the different factors evaluated by examiners ( 1 and 2$)$ was interpreted by a Kappa interrater coefficient. The actual diagnoses made by examiners were compared by a Kappa statistic, and the results were shown according to the actual clinical condition or final diagnosis.

\section{Results}

Forty-four bone specimens resected from each bone disease comprising 22 cases of ORNJ, 6 cases of OMJ, and 16 cases of MRONJ were included. Thickness and percentage of bone tissue and connective tissue varied considerably, as the specimens were obtained from incisional biopsies. Minimum thickness was $5 \mathrm{~mm}$. All specimens had the ideal thickness required for diagnosis.

The demographic data are displayed in Table 2 The concordance between the different factors evaluated by examiners (1 and 2) was interpreted by a Kappa interrater coefficient, which was equal to 1 , that is, there was no interrater difference in the evaluation of the presence or absence of each variable. Most of the analyzed criteria were detected in MRONJ, ORNJ, and OMJ, as shown in Table 1, resulting in no statistically significant differences in their prevalence rates. The percentages

Table 2. Table showing incidence rates by gender, age, and lesion sites.

\begin{tabular}{lccc}
\hline Bone pathologies & Gender & Age (years) & Site \\
\hline 6 OMJ samples & 5 Males & - & - \\
& 1 Females & $\begin{array}{c}53 \text { to } 56 \\
\text { (mean: } 54.3)\end{array}$ & 6 Mandible \\
& 4 Males & - & - \\
22 MRONJ & 18 Females & $\begin{array}{c}43 \text { to } 89 \\
\text { (mean: } 58.5)\end{array}$ & 19 Mandible \\
& & - & 3 Maxilla \\
& 15 Males & - & - \\
16 ORN samples & 1 Females & $\begin{array}{c}42 \text { to } 75 \\
\text { (mean: } 58.1)\end{array}$ & 16 Mandible \\
\hline
\end{tabular}

of all evaluated structures are described in Table 3 , including general aspects of bone tissue, such as necrotic $(p=0.211)$ and reactive bone $(p=0.723)$, along with osteoclastic $(p=0.146)$ and osteoblastic cells $(p=0.432)$. In addition, the rate inflammation $(p=0.672)$, especially chronic, was the same for all the three diseases, considering lymphocytes $(p=0.054)$ and macrophages $(p=0.490)$. Also, the presence of blood vessels $(p=0.870)$ and hyperemia and thrombosis $(p=0.472)$ was similar. Another common finding was the presence of biofilm-forming microorganisms $(p=0.160)$ (Table 3$)$.

Significant differences were detected in empty osteocyte lacunae, which were less frequent in ORNJ $(p=0.042)$ than in MRONJ and OMJ, and neutrophils, which were less frequent in MRONJ $(p<0.05)$ than in ORNJ and OMJ (Table 3).

There was no consistent histopathological diagnosis between examiners (Kappa $=0.23$ ). The tentative diagnosis made by each examiner is described in Table 4.

\section{Discussion}

This study aimed to evaluate the histopathological aspects of OMJ, ORNJ, and MRONJ considering close

Table 3. Table showing the percentage of all variables compared among the diseases.

\begin{tabular}{lcccc}
\hline Variable & OMJ & MRONJ & ORNJ & p-value \\
\hline Necrotic bone & 83.3 & 93.7 & 100 & 0.211 \\
Osteoclast & 33.3 & 18.7 & 50 & 0.146 \\
Reactive bone & 50 & 31.2 & 36.6 & 0.723 \\
Osteoblastic coating & 50 & 31.2 & 22.7 & 0.432 \\
Empty osteocyte lacunae & 83.3 & 100 & 22.7 & 0.042 \\
\hline Inflammation & 83.3 & 75 & 86.6 & 0.672 \\
\hline Lymphocytes & 66.6 & 43.7 & 81.8 & 0.054 \\
Macrophages & 50 & 31.2 & 50 & 0,490 \\
Neutrophils & 83.3 & 12.5 & 86.3 & $\leq 0.001$ \\
\hline Blood vessels & 66.6 & 56.2 & 54.5 & 0.870 \\
\hline Hyperemia and thrombosis & 50 & 43.7 & 63.3 & 0.472 \\
\hline Microorganisms & 50 & 87.5 & 100 & 0.160 \\
\hline
\end{tabular}


Table 4. Tentative diagnosis of each examiner per sample, indicating lack of consistency between them (Kappa=0.23).

\begin{tabular}{|c|c|c|}
\hline Examiner 1 & Examiner 2 & Final Diagnosis \\
\hline OM & OMMBF & ORN \\
\hline OM & OM & ORN \\
\hline OMMBF & $O M$ & ORN \\
\hline OM & $O M$ & ORN \\
\hline OMMBF & OMMBF & ORN \\
\hline OMMBF & $O M$ & ORN \\
\hline OMMBF & ORN & ORN \\
\hline OMMBF & ORN & ORN \\
\hline OM & $\mathrm{OMMBF}$ & ORN \\
\hline OMMBF & OMMBF & ORN \\
\hline OMMBF & OMMBF & ORN \\
\hline OM & $\mathrm{OM}$ & ORN \\
\hline OM & ORN & ORN \\
\hline OM & $O M$ & ORN \\
\hline OM & $O M$ & ORN \\
\hline OM & $O M$ & ORN \\
\hline ORN & ORN & ORN \\
\hline OM & $\mathrm{OM}$ & ORN \\
\hline$O M$ & $O M$ & ORN \\
\hline OM & ORN & ORN \\
\hline$O M$ & OMMBF & ORN \\
\hline OM & ORN & ORN \\
\hline OM & $O M$ & OM \\
\hline OM & ORN & OM \\
\hline ORN & ORN & OM \\
\hline OMMBF & ORN & OM \\
\hline OMMBF & OMMBF & OM \\
\hline OM & ORN & OM \\
\hline OMMBF & ORN & OMMBF \\
\hline OM & OMMBF & OMMBF \\
\hline ORN & OMMBF & OMMBF \\
\hline OM & OMMBF & OMMBF \\
\hline OM & ORN & OMMBF \\
\hline$O M$ & OM & OMMBF \\
\hline OM & OMMBF & OMMBF \\
\hline OM & ORN & OMMBF \\
\hline OMMBF & OMMBF & OMMBF \\
\hline OM & $O M$ & $\mathrm{OMMBF}$ \\
\hline ORN & ORN & OMMBF \\
\hline OM & ORN & OMMBF \\
\hline OM & $O M$ & OMMBF \\
\hline OMMBF & OMMBF & OMMBF \\
\hline$O M$ & OMMBF & OMMBF \\
\hline OM & ORN & OMMBF \\
\hline
\end{tabular}

similarities among them and, consequently, to make clinicians aware of the importance of understanding the etiopathogenesis and clinical manifestations of each disease. Few studies with the same objectives were found in a recent review of the literature, ${ }^{1,3,10,11}$ but more information was needed in order to clarify histological differences between the diseases.

It is important to emphasize that the pathologies chosen in the present study had already been diagnosed based upon the patients' clinical information given by the clinicians. Since the information was retrieved from the files of an oral pathology service, the number of cases was not identical, but all cases were maintained in the study, thus avoiding the significant reduction of sample size to a minimum. Another important aspect refers to OMJ. It is true that MRONJ and ORNJ can easily evolve into osteomyelitis once secondary infection occurs, ${ }^{12}$ which can hinder the final diagnosis as well as the comprehension of the etiological process of each disease. However, according to the patients' records, only those cases originating from chronic or acute dentoalveolar abscesses were chosen, with no association with radiation therapy or bisphosphonates, or with any other antiresorptive drug.

The analysis of the histological criteria was based on their presence or not, and no morphometric method was used to quantify cells or structures, as this is not a routine practice for the analyzed diseases. Nevertheless, most of the analyzed criteria did not reveal significant differences, but it was clear that some of them were more prevalent in one or other disease. These aspects will also be discussed in order to elucidate some subtleties of each disease. Also, regarding the methodology, the morphometric analysis was not performed mainly due to the large variability in sample size, since the study was performed with biopsy specimens from different patients performed by different surgeons.

The presence of necrotic bone was fairly common among the clinical diseases. Marx and Tursun ${ }^{2}$ identified necrotic bone in 100 of all OMJ, ORNJ, and MRONJ specimens, considering a set of characteristics such as the presence of empty osteocyte lacunae, absence of osteoblastic rimming, and empty Haversian and Volkmann canals. Although these characteristics were individually analyzed in our study, they were 
also taken as characteristics that compounded the general picture of bone necrosis.

Although there was no significant difference in the presence of osteoclasts among the three clinical diseases, it was clear that they were lower in MRONJ specimens than in the other diseases, possibly due to the mechanism of action of the drug involved in the osteonecrotic process. Interestingly, in the study of Marx and Tursun, ${ }^{2}$ osteoclasts were seen only in OMJ specimens. The same occurred in reactive bone and osteoblastic coating. Despite the lack of significant difference among the diseases, OMJ specimens showed larger amounts of both criteria.

When inflammation was assessed, more than 75 of the samples revealed presence of some kind of leukocytes, and neutrophils were more frequent in OMJ (83.3) and ORNJ specimens (86.3). Significant lack of leukocytes was detected in MRONJ, in agreement with Marx and Tursun. ${ }^{2}$

Compromised vascularization was expected to be seen, especially in ORNJ and MRONJ, given the etiological factors of both diseases. When the diseases were compared, no significant differences were detected. Hyperemia and thrombosis predominated in ORNJ (63.3), followed by OMJ (50) and MRONJ (43.7). There was absence of blood vessels in MRONJ samples. ${ }^{10}$

Microorganisms were found in many samples, revealing no significant differences among the diseases. In OMJ, microorganisms were observed in 50 of the samples, compared to 87.5 and 100 in ORNJ and MRONJ, respectively. Microorganisms

\section{References}

1. Guimarães EP, Pedreira FR, Jham BC, Carli ML, Pereira AA, Hanemann JA. Clinical management of suppurative osteomyelitis, bisphosphonate-related osteonecrosis, and osteoradionecrosis: report of three cases and review of the literature. Case Rep Dent. 2013;2013:402096. https://doi.org/10.1155/2013/402096

2. Marx RE, Tursun R. Suppurative osteomyelitis, bisphosphonate induced osteonecrosis, osteoradionecrosis: a blinded histopathologic comparison and its implications for the mechanism of each disease. Int J Oral Maxillofac Surg. 2012 Mar;41(3):283-9. https://doi.org/10.1016/j.ijom.2011.12.016 of the genus Actinomyces have been found in these disease by several authors, ${ }^{6,13,14,15,16}$ including by Marx and Tursun ${ }^{2}$ The microorganisms were detected on trabecular bone surfaces in 76 of MRONJ and in 58 of ORNJ cases. In OMJ samples, these microorganisms were found in medullary spaces. ${ }^{2}$ On the other hand, in our study, OMJ samples showed a lower amount of microorganisms, compared to the other samples. It is important to emphasize that only 6 OMJ samples were evaluated in the present study.

Another aim of this study was to test oral pathologists' capacity to establish a histopathological diagnosis of the proposed diseases in the absence of clinical information. Pathologists who work with diagnoses and know the three entities know that anamnesis is crucial for the diagnosis, given that clinical and radiographic features are very similar.

With regard to the first research question, the blinded evaluation by two oral pathologists confirmed the hypothesis that it is not possible to establish a final diagnosis only by way of microscopic analysis. The diagnoses of the diseases mentioned above tended to be quite similar microscopically, as unanimously endorsed by experts.

\section{Conclusions}

The histopathological aspects of ORNJ, OMJ, and MRONJ do not permit a conclusive diagnosis, emphasizing the necessity of a detailed clinical report and of clinicians' expertise.
3. Mitsimponas KT, Moebius P, Amann K, Stockmann P, Schlegel KA, Neukam FW et al. Osteo-radio-necrosis (ORN) and bisphosphonate-related osteonecrosis of the jaws (BRONJ): the histopathological differences under the clinical similarities. Int J Clin Exp Pathol. 2014 Jan;7(2):496-508.

4. Lew DP, Waldvogel FA. Osteomyelitis. Lancet 2004;364(9431):369-79. https://doi.org/10.1016/S0140-6736(04)16727-5

5. Marx RE. Osteoradionecrosis: a new concept of its pathophysiology. J Oral Maxillofac Surg. 1983 May;41(5):2838. https://doi.org/10.1016/0278-2391(83)90294-X 
6. Ruggiero SL, Dodson TB, Fantasia J, Goodday R, Aghaloo T, Mehrotra B et al. American Association of Oral and Maxillofacial Surgeons position paper on medication-related osteonecrosis of the jaw: 2014 update. J Oral Maxillofac Surg. 2014 Oct;72(10):1938-56. https://doi.org/10.1016/i.joms.2014.04.031

7. Obinata K, Shirai S, Ito H, Nakamura M, Carrozzo M, Macleod I et al. Image findings of bisphosphonate related osteonecrosis of jaws comparing with osteoradionecrosis. Dentomaxillofac Radiol. 2017 Jul;46(5):20160281. https://doi.org/10.1259/dmfr.20160281

8. Cardoso CL, Barros CA, Curra C, Fernandes LM, Franzolin $\mathrm{SO}$, Ferreira Júnior JS et al. Radiographic findings in patients with medication-related osteonecrosis of the jaw. Int J Dent. 2017;2017:3190301. https://doi.org/10.1155/2017/3190301

9. Curi MM, Cardoso CL, Lima HG, Kowalski LP, Martins MD. Histopathologic and histomorphometric analysis of irradiation injury in bone and the surrounding soft tissues of the jaws. J Oral Maxillofac Surg. 2016 Jan;74(1):190-9. https://doi.org/10.1016/j.joms.2015.07.009

10. Hansen T, Kunkel M, Weber A, James Kirkpatrick C. Osteonecrosis of the jaws in patients treated with bisphosphonates - histomorphologic analysis in comparison with infected osteoradionecrosis. J Oral Pathol Med. 2006 Mar;35(3):155-60. https://doi.org/10.1111/j.1600-0714.2006.00391.x
11. Carmagnola D, Canciani E, Sozzi D, Biglioli F, Moneghini L, Dellavia C. Histological findings on jaw osteonecrosis associated with bisphosphonates (BONJ) or with radiotherapy (ORN) in humans. Acta Odontol Scand. 2013 Nov; 71(6):1410-7. https://doi.org/10.3109/00016357.2013.765592

12. Tardast A, Sjöman R, Løes S, Abtahi J. Bisphosphonate associated osteomyelitis of the jaw in patients with bony exposure: prevention, a new way of thinking. J Appl Oral Sci. 2015 May-Jun;23(3):310-4. https://doi.org/10.1590/1678-775720140506 PMID:26221926

13. Aftimos V, Zeinoun T, Bou Tayeh R, Aftimos G. Bisphosphonate related osteonecrosis of the jaw: a study of 18 cases associated with fungal infection. Int J Dent. 2014;2014:869067. https://doi.org/10.1155/2014/869067 PMID:24696686

14. Boff RC, Salum FG, Figueiredo MA, Cherubini K. Important aspects regarding the role of microorganisms in bisphosphonate-related osteonecrosis of the jaws. Arch Oral Biol. 2014 Aug;59(8):790-9. https://doi.org/10.1016/i.archoralbio.2014.05.002

15. Curi MM, Dib LL, Kowalski LP, Landman G, Mangini C. Opportunistic actinomycosis in osteoradionecrosis of the jaws in patients affected by head and neck cancer: incidence and clinical significance. Oral Oncol. 2000 May;36(3):294-9. https://doi.org/10.1016/S1368-8375(99)00080-9

16. De Ceulaer J, Tacconelli E, Vandecasteele SJ. Actinomyces osteomyelitis in bisphosphonate-related osteonecrosis of the jaw (BRONJ): the missing link? Eur J Clin Microbiol Infect Dis. 2014 Nov;33(11):1873-80. https://doi.org/10.1007/s10096-014-2160-5 